\title{
Hypothalamus-pituitary-adrenal (HPA) axis, chronic stress, hair cortisol, metabolic syndrome and mindfulness
}

\author{
Helen Patricia Gaete* \\ Ferry View Health Centre, 25/27 John Wilson Street, Woolwich, London SE18 6 PZ, UK
}

\section{Background information}

Over the past 60 years, there has been an exponential expansion in the field of neuroendocrinology. The HPA Axis (Hypothalamus Pituitary Adrenal Axis) is affected by both internal physiological stressors (cytokines, hypoxia, macromolecules etc) and by external stressors (source of anxiety, fear etc.) which threaten the organisms homeostasis. Cortisol binds to cortisol receptors throughout the body. The hormone receptor complex is to be found in the nucleus of the cells, where it binds to DNA (docking) (Figure 3).The consequences of this bond vary considerably. In some cells cortisol can induce some genes and repress others. In cases where the HPA Axis is overactive the amount of cortisol in the nucleus of the cells may increase by approximately tenfold. There is good evidence over the past years of dysfunction of the HPA Axis in Major Depression. Nemeroff [1] has suggested that the assessment of the HPA Axis activity may be a useful tool in clinical practice. Prof. Nemeroff [1], has emphasised the opportunity of "early intervention in at risk individuals". Prof. Nemeroff suggests that individuals who have been exposed to untoward early life stress can be considered an "at risk population" [1]. There is emerging evidence showing the usefulness of Hair Cortisol analysis as an indicator of sub-acute and chronic stress [2]. Can we use Hair Cortisol levels prophylactically in Primary Care to assess HPA Axis activity in individuals at risk? Can we use Hair Cortisol to monitor response to treatment in clinical practice in an effort to provide more effective personalised care?

\section{Chronic stress and HPA axis}

Cortisol plays an important role as a regulator and modulator of

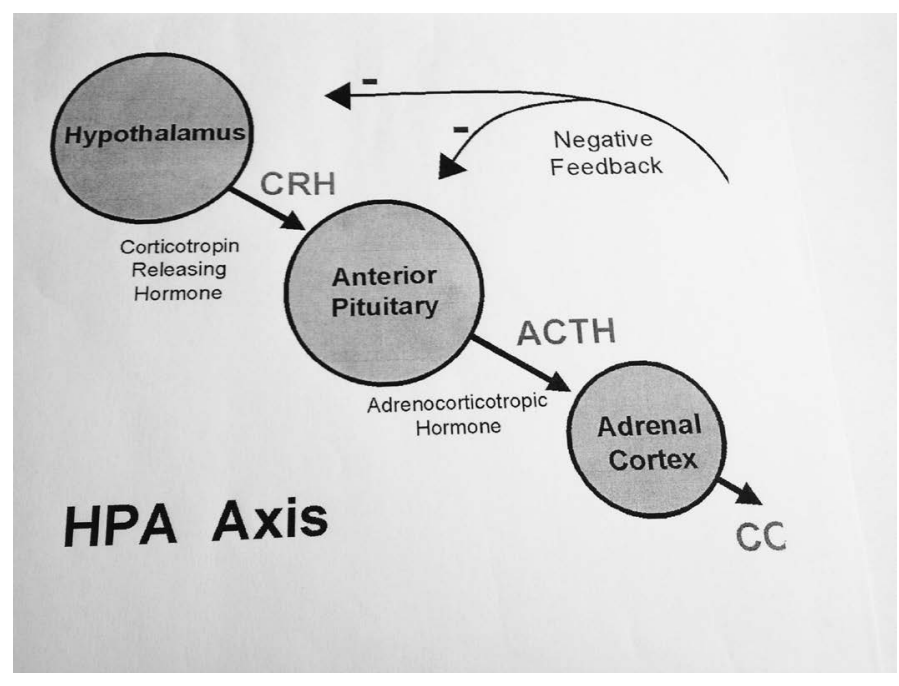

Figure 1. HPA Axis. intermediate metabolism, and also of the immune system. It affects the secretion of ACTH and CRH via the hypothalamus-pituitary feedback system. The three hormones $\mathrm{CRH}, \mathrm{ACTH}$ and Cortisol form a regulatory system with feed-back inhibition in order to adjust the plasma cortisol level to the respective requirements. The activation of the HPA Axis during stress affects all the systems in the body. The HPA Axis presents a "common Pathway".

There seems to be a gap between the individual experiencing trauma/other severe stressors (significant losses, abuse, bullying, neglect, other) and the emergence of clinical symptoms. Between the activation of the HPA Axis in chronic stress and the onset of psychopathology. This provides "a window of opportunity" for early detection of dysregulation, assessment and early intervention.

\section{Hair cortisol a biomarker of chronic stress}

Van Uum et al. [2] mention that Hair Cortisol levels are being increasingly used in a variety of pathological situations, because it can provide a long term measure of systemic cortisol exposure. Dettenborn et al. [3], used Hair Cortisol analysis to rate levels of psychological stress. Individuals who had been unemployed for at least a year, were compared with currently employed control subjects. Cortisol concentrations were significantly higher in the unemployed group.

\section{Chronic stress, HPA Axis, depression and suicidality}

Sigmund Freud in his manuscript "Mourning and Melancholia" highlighted the importance of early loss on vulnerability to depression in adulthood, particularly in response to loss experienced in adulthood. The study by Agid et al. [4], showed in their case control study that the rate of parental loss in the depressed patient group was $29.1 \%$, in the bipolar group was $17.7 \%$ and in the schizophrenia group was $22.4 \%$, compared to rates of $7.6-7.9 \%$ in the control subjects.

The biological vulnerability seems to be mediated, at least in part, by persistent activation and hyper responsiveness of the hypothalamic and extra hypothalamic corticotrophin-releasing factor (CRF) circuits,

Correspondence to: Helen Patricia Gaete, Ferry View Health Centre, 25/27 John Wilson Street, Woolwich, London SE18 6 PZ, UK, Email: patriciagaete@hotmail.co.uk

Key words: hypothalamus-pituitary-adrenal (hpa) axis, corticotrophic releasing hormone (crh), sympathetic-adrenal-medullary (sam) axis,chronic stress, hair cortisol levels, early life stress (els), "at risk individuals", dexamethasone suppression test (dst), depression, metabolic syndrome (mets), 11-beta hydroxysteroid dehydrogenase, mindfulness

Received: September 26, 2016; Accepted: October 03, 2016; Published: October 05, 2016 


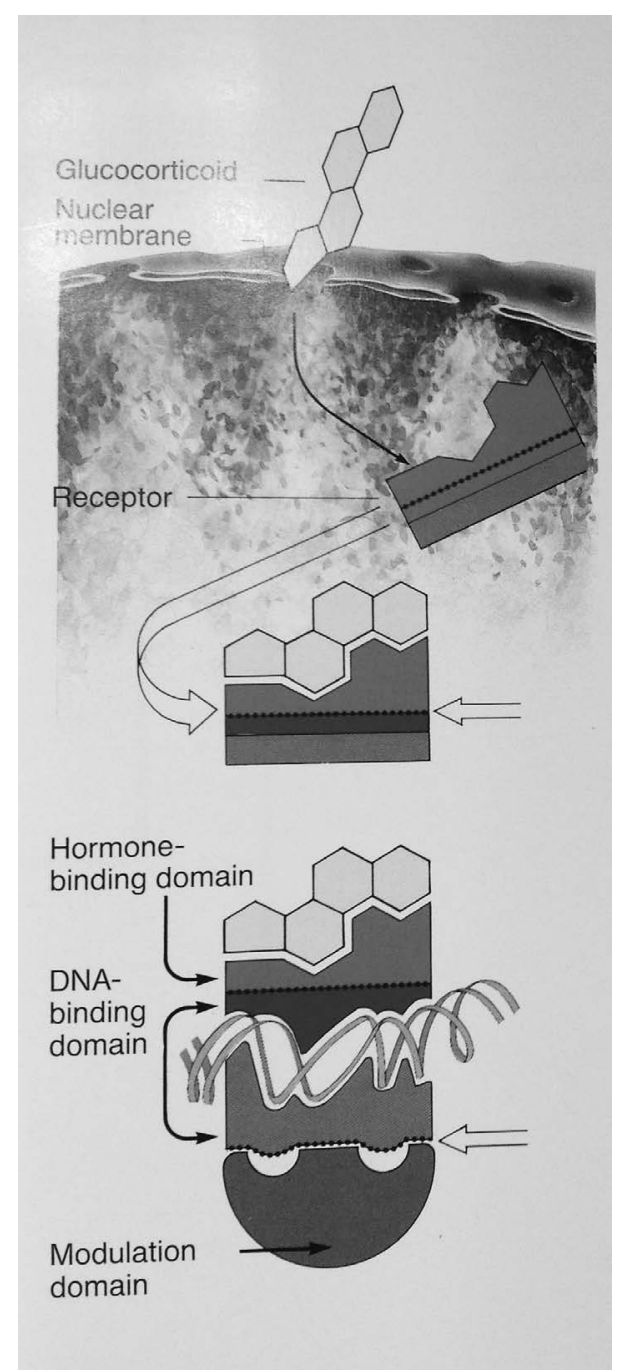

Figure 2. Scheme of the interactionof a steroid hormone with its specifi c receptor and shown in studies by Plotsky, Meaney and Nemeroff in rats. Nemeroff [1] completed a pilot clinical study that demonstrated that women with a history of child abuse with or without major depression, presented hyperactivity of the HPA Axis in response to a social stressor (Trier Social Stress Test).

Sachar et al. documented the link between the stress hormone hypersecretion and affective disorders. An excess of cortisol, and DST suppression have been reported for a long time in patients with mood disorder. Major Depression is associated with dysfunction of the HPA Axis. There is activation of the HPA Axis and also blunting of the normal diurnal cortisol profiles.

Aihara et al. [5], found that in patients with Major Depressive Disorder, enhanced cortisol normalised after successful pharmacotherapy. The results indicated that depressed patients remitted with antidepressant medication, presenting resolution of HPA dysregulation and alteration of regional glucose metabolism in the prefrontal cortical, limbic and paralimbic areas. Jokinen et al. [6], studied the predictive value of DST for suicide in a group of depressed patients with and without an index suicide attempt. They found that the non-suppressor status was significantly associated with suicide, indicating that HPA Axis hyperactivity is a risk factor for suicide in this group.

\section{Early life stress}

New evidence has emerged on early life stress induced metabolic derangements. Early life stress can alter the expression of the genes in peripheral tissues, such as glucocorticoid receptor and 11- beta hydroxysteroid dehydrogenase. Morris et al. [7] proposes that interactions between altered HPA Axis activity and liver 11-beta hydrogenase modulates both tissue and circulating glucocorticoid availability, with adverse metabolic consequences

\section{Chronic stress, HPA Axis \& metabolic abnormalities}

The Metabolic Syndrome involves a cluster of cardio metabolic abnormalities, including hypertension, abdominal obesity,

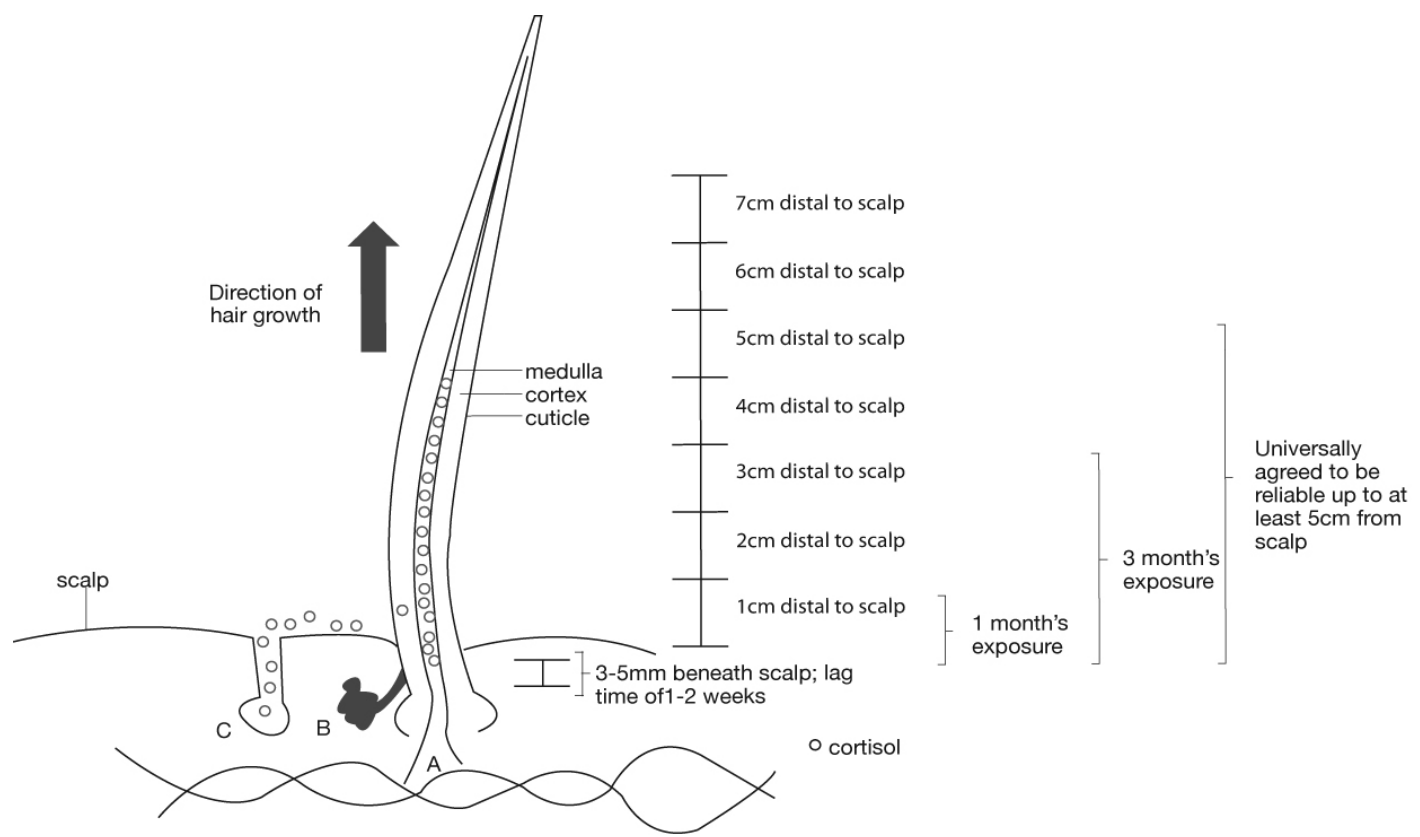

Figure 3. Proposed mechanisms for incorporation of cortisol into hair via blood (A), sebum (B), and sweat (C). 


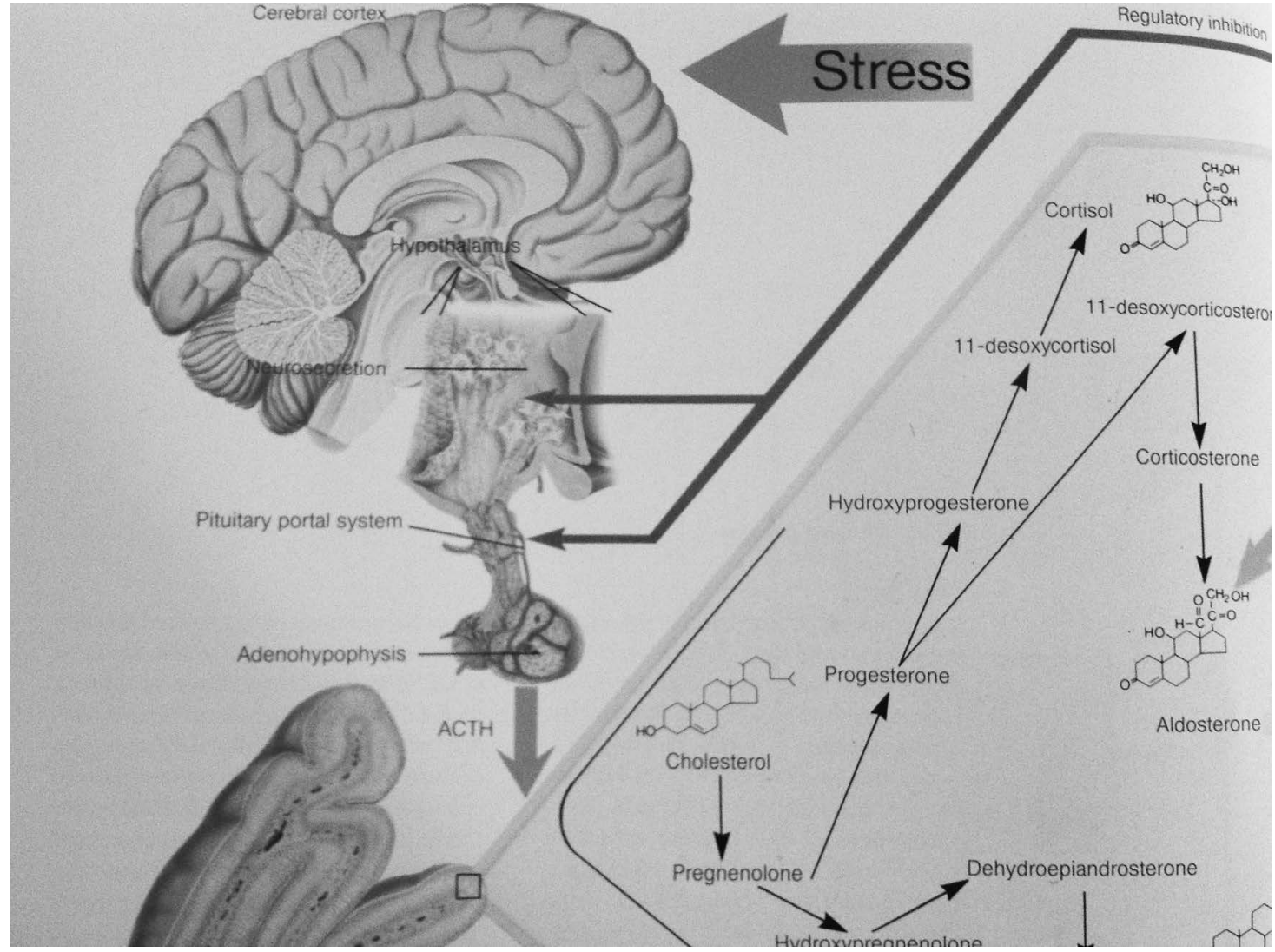

Figure 4. Cortisol production during stress (Breckwoldt, Neumann, Brauer, "Exempla Endocrinologica,1994).

hyperglycaemia and dyslipidaemia. It has been suggested that increased activity of the HPA Axis, with increased levels of glucocorticoid hormones may contribute to the development of MetS [8]. Also, a normal physiological difference in long term cortisol secretion, assessed through Hair Cortisol levels, shows a relevant relationship with MetS [9].

New evidence has emerged on early life stress induced metabolic derangements (eg. hyperinsulinemia and altered insulin sensitivity on exposure to a high energy diet later on in life). Vogelzangs et al. [10] suggested that there is a synergistic relationship between depression, cortisol and the Metabolic Syndrome.

\section{Modulation of the HPA axis}

Research has shown that the HPA Axis can be modulated by treating the underlying condition, achieving healthier weight, improving length and quality of sleep, increasing slow wave phase. Social support and Mindfulness can also help to modulate the HPA Axis. Stalder et al. have shown the beneficial effects on metabolism of small changes in long term secretion of cortisol [9].

\section{Sleep and HPA axis}

At times of Stress, amygdala glucocorticoid receptors (GRs) may be preferentially activated, increasing $\mathrm{CRH}$. Elevated $\mathrm{CRH}$ increases Electroencephalogram (EEG) frequency, decreasing Slow Wave Sleep (SWS), increasing light sleep and wakefulness.

On the other hand, sleep disturbance (insomnia, sleep apnoea), can aggravate HPA Axis dysfunction. In the case of Obstructive sleep apnoea, every time patient gasps for breath at night, there is pulsatile cortisol release and autonomic activation. However, when an individual is able to achieve a better quality of sleep, the slow wave phase (SWS) can suppress the HPA Axis. Helping patients to improve their quality of sleep, can help them modulate their HPA Axis. This would have beneficial metabolic effects. It would also help them to be better able to respond to treatment.

\section{HPA Axis and mindfulness}

Brunner et al. [11], through their case-control study, provided evidence that Chronic Stress may be a cause of the Metabolic Syndrome (MetS). Using Whitchall II Cohort, Brunner et al. showed that Metabolic Syndrome was associated with 24 hour cortisol metabolite and normetanephrine output and with cardiac autonomic activity [11] Differences in cortisol output and cardiac autonomic activity associated with Metabolic Syndrome were reduced in ex-cases, indicating that the changes are potentially reversible. Psychosocial factors explained in part the increased normetanephrine output associated with the Metabolic Syndrome. Adverse cardiac autonomic functions related to the syndrome were attributable both to psychosocial factors and degree of obesity. The Metabolic Syndrome may be an intermediate between long term psychosocial stress and coronary disease. Markers of inflammation predict weight gain, Diabetes and Chronic Heart Disease. The longitudinal component of the study provides evidence for the reversibility of the neuroendocrine and inflammatory alterations linked with the Metabolic Syndrome.

Cresswell et al. [12] have proposed an evidence-based biological model of mindfulness, stress buffering and health. They propose a conceptual model of the biological pathways linking Mindfulness and stress-buffering. This account suggests that Mindfulness training might 
alter neural stress-processing dynamics in high stress participants, reduce SAM Axis or HPA Axis reactivity.

Cresswell et al. [12] propose that Mindfulness can have a stress buffering effect. Well controlled studies have suggested that Mindfulness-training interventions can improve a broad range of mental and physical health outcomes (e.g. HIV pathogenesis, depression relapse, inflammation).

They have offered an evidence based biological model of mindfulness. This suggests that mindfulness may alter neural stressprocessing dynamics. In high stress participants, Mindfulness can reduce SAM (Sympathetic-adrenal medullary) or HPA Axis reactivity and help normalize dysregulated stress signalling in these systems.

\section{Conclusions}

There is increasing evidence of long-term neurobiological consequences of Chronic Stress. There seems to be a gap between the over activation of the HPA Axis and the emergence of symptoms and the different syndromes. Evidence has shown that persistently raised levels of Cortisol can lead to metabolic abnormalities, and are linked with conditions like depression. The HPA Axis is fundamentally a dynamic system. It changes in time. Could we use Hair Cortisol to do longitudinal studies?

Could we identify the "at risk population", monitor them and intervene early by helping individuals to develop protective factors, to modulate the HPA axis and reduce risk of metabolic abnormalities and of development of psychopathology? In clinical practice we are aware of the value of the protective factors (social support, mindfulness training, counselling, developing skills in resilience, etc.). Could we intervene early in Primary Care to protect the "patients at risk" and also patients who are developing early symptoms of mental health problems? Would it be useful to look at the national figures of the prevalence of child abuse, neglect, significant losses, trauma and the prevalence of psychiatric disorders?

Would it be helpful to have an Agenda to improve awareness on how to manage Chronic Stress in schools as part of the syllabus?

Also, more awareness on the implications of Chronic Stress (bullying, abuse, neglect, etc), and to have more resources for Pastoral Care and Counselling available in schools and universities? It would help to have more counselling facilities, training in Mindfulness and workshops available to the Public, to Primary, Secondary and Terciary Care. In view of the current evidence, a prophylactic and proactive approach would seem reasonable to improve the overall health of the nation, reducing morbidity and complications. This could constitute a very cost-effective strategy long term.

\section{Going forward}

Can we use Hair Cortisol levels to do longitudinal studies, to better understand the dynamics of the HPA Axis and SAM axis in time? To understand how long does it take between the activation of the HPA Axis and the first signs and symptoms of pathology (medical/psychological)? It would be interesting to study the HPA Axis, using Hair Cortisol levels in "vulnerable individuals" before and after Mindfulness training and compare to controlled group. Also, measuring the cardio metabolic parameters.

\section{References}

1. Nemeroff CB (1999) "The pre-eminent role of untoward experience on vulnerability to major psychiatric disorders: the nature-nurture controversy revisited and soon to be resolved". Mol Psychiatry 4: 106-108. [Crossref]

2. Van Uum S, Gow R, Koren G, Rieder M (2011) Hair cortisol content in patients with adrenal insufficiency on hydrocortisone replacement therapy. Clin Endocrinol (Oxf) 74: 687-93. [Crossref]

3. Dettenborn L, Tietze A, Bruckner F, Kirschbaum C (2010) Higher cortiso content in hair among long-term unemployed individuals compared to controls. Psychoneuroendocrinology 35: 1404-9 [Crossref]

4. Agid O, Shapira B, Zislin J, Ritsner M, Hanin B, et al. (1999) "Environment and vulnerability to major psychiatric illness: a case control study of early parental loss in major depression, bipolar disorder and schizophrenia". Mol Psychiatry 4: 163-172. [Crossref]

5. Aihara M, Ida I, Yuuki N, Oshima A, Kumano H, et al. (2007) HPA axis dysfunction in unmedicated major depressive disorder and its normalization by pharmacotherapy correlates with alteration of neural activity in prefrontal cortex and limbic/paralimbic regions. Psychiatry Res 155: 245-56. [Crossref]

6. Jokinen J, Carlborg A, Martensson B, Forslund K, Nordström AL, et al. (2007) DST non-suppression predicts suicide after attempted suicide. Psychiatry Res 150: 297-303. [Crossref]

7. Morris MJ, Maniam J, Antoniadis C (2014) Early-Life Stress, HPA Axis Adaptation, and Mechanisms Contributing to Later Health Outcomes. Front Endocrinol (Lausanne) 5: 73. [Crossref]

8. Friedman TC, Mastorakos G, Newman TD, Mullen NM, Horton EG, et al (1996)"Carbohydrate and lipid metabolism in endogenous hypercortisolism: shared features with metabolic syndrome X and NIDDM". Endocr J 43: 645-655. [Crossref]

9. Stalder T, Kirschbaum C, Alexander N, Bornstein SR, Gao W, et al. (2013) Cortisol in hair and the metabolic syndrome. J Clin Endocrinol Metab 98: 2573-2580. [Crossref]

10. Vogelzangs N, Suthers K, Ferrucci L, Simonsick EM, Ble A, et al. (2007) Hypercortisolemic depression is associated with the metabolic syndrome in late-life. Psychoneuroendocrinology 32: 151-9. [Crossref]

11. Brunner EJ, Hemingway H, Walker BR, Page M, Clarke P, et al. (2002) Adrenocortical, autonomic, and inflammatory causes of the metabolic syndrome: nested case-control study. Circulation 106(21): 2659-65. [Crossref]

12. Cresswell JD, Lindsay EK (2014) How Does Mindfulness Training Affect Health? A Mindfulness Stress Buffering Account. Psycho Sci 23: 401-407.

Copyright: (C2016 Gaete HP. This is an open-access article distributed under the terms of the Creative Commons Attribution License, which permits unrestricted use, distribution, and reproduction in any medium, provided the original author and source are credited. 